\title{
Image Segmentation Analysis based on Maximum Entropy Algorithm Chen Rong
}

\author{
Department of Physics, Nanchang Normal University,Nanchang, China \\ e-mail: xinsea@163.com
}

Keywords:image; segmentation; analysis; algorithm; maximum entropy;

\begin{abstract}
This paper introduces the definition of maximum entropy,the principle of onedimensional and 2-D maximum entropy. This paper solves the image segmentation problem by using the maximum entropy algorithm. The image gray value is divided into two regions of the background and the object.The key problem associated with this method is to find the optimal parameter extracting objects from background, the proposed method is used to segment image and ideal segmentation results can be obtained with less computation cost.Experimental simulation shows that the algorithm has a higher accuracy while preserving the edge infomation,and maximum entropy has been proven to be an efficient method for image segmentation.
\end{abstract}

\section{Introduction}

Processing method of image segmentation contains many ways as follow: basing on threshold segmentation,basing on the entropy and the histogram, basing on the method of Otsu segmentation, basing on a grayscale arithmetic mean, basing on boundary extraction segmentation, basing on region segmentation. In order to improve the effect of image segmentation,in this paper, the best threshold is obtained based on maximum entropy principle.

\section{The Definition of Maximum Entropy}

The amount of information is related to the probability of the event. The smaller the probability of event occurring is, the greater the amount of information is. If two events are independent, the amount of information they contain is equal to the amount of information contained in their respective, and called the information additivity.

The information content measure should be:

$$
I(X=x)=-\log p(x)
$$

The smaller $p(x)$ is, the greater the uncertainty of events $[X=x]$ is. Information measure is the measure of uncertainty of the event.

The amount of information events are written as:

$$
I\left(x_{i}\right)=-\log p\left(x_{i}\right)
$$

For the average value of ${ }^{I\left(x_{i}\right)}$, get the entropy of $X$,entropy function is as follows:

$$
\begin{aligned}
& H(X)=E\left[I\left(x_{i}\right)\right]=E\left[-\log p\left(x_{i}\right)\right] \\
& =-\sum_{i=1}^{n} p\left(x_{i}\right) \log p\left(x_{i}\right)
\end{aligned}
$$

$H(X)$ is the average amount of information source

$\left\{X, P_{X}(\cdot)\right\}$. $H(X)$ reached a maximum at $\log |X|$ when all $|X|$ value is equal to the probability that $X$ occur,and the $|X|$ is the number of elements in the collection of $X$.

$$
\left.H(X)\right|_{p_{X}(x)=\frac{1}{|X|}}-H(X)=-\sum_{x} \frac{1}{|X|} \log \frac{1}{|X|}+\sum_{x} p(x) \log p(x) \quad=-\sum_{x} p(x) \log \frac{1}{|X| p(x)}
$$




$$
\geq \sum_{x} p(x)\left[1-\frac{1}{|X| p(x)}\right]=0
$$

That is:

$$
\left.H(X)\right|_{p_{X}(x)=\frac{1}{|X|}} \geq H(X) \text {. When } p_{X}(x)=\frac{1}{|X|}, H(X) \text { is the maximum value. }
$$

\section{The Principle of One-dimensional Maximum Entropy}

Digital image is composed of pixels, each pixel has a certain gray value, the gray scale range of image is $0 \sim 255$. The total number gray value of all pixels is the resolution of the image, and the number of pixels of each gray value in different images is uncertain. That is,the gray value of the pixel is random, so the probability of each gray which appears is as random variables. Entropy is a concept of information measurement, its principle is through the gray-scale histogram of each pixel for the analysis of digital image[1].The gray level of pixels are divided into L grade, the number of pixels for each gray level is $\mathrm{Ni}$, the probability of occurrence for each gray level is as follows:

$$
\begin{aligned}
& p_{i}= \frac{N_{i}}{N} \\
& \sum_{i=0}^{L-1} p_{i}=1
\end{aligned}
$$

In the type(5):Ni is the total number of pixels for i grade; pi is the probability of the pixels for i grade, pi is to reflect the change in gray level of pixels[2]. In the type(6): $\mathrm{L}=256$, the image gray level is from 0 to 255, is to be divided into 256 levels. Grayscale value is 0 , stands for the black, gray value is 255, stands for the white.

Distribution of image boundary is uncertain,the entropy at the junction of the target and background in the image is the maximum entropy ,that is maximum information entropy, reflects the contour of the image. The threshold is set to t, the gray image is higher than $t$, constitute the region of $\mathrm{O}$, less than $\mathrm{t}$, constitute the background region of $\mathrm{B}$.

Probability distribution of the two kinds of regions are as follows:

The target region of $\mathrm{O}$ :

$\frac{p_{i}}{p_{o}} \quad i=0,1,2, \cdots, t$

The background region of $\mathrm{B}$ :

$\frac{p_{i}}{p_{b}} \quad i=t+1, t+2, \cdots, L-1$

The basic idea of maximum entropy method: the probability of target region of $\mathrm{O}$ and background region of $\mathrm{B}$ are as follows.

$$
\begin{gathered}
p_{o}=\sum_{i=0}^{t} p_{i} \\
p_{b}=\sum_{i=t+1}^{L-1} p_{i} \\
p_{o}+p_{b}=1 \\
H_{o}=-\sum_{i=0}^{t} \frac{p_{i}}{p_{o}} \log \frac{p_{i}}{p_{o}} \\
H_{b}=-\sum_{i=t+1}^{L-1} \frac{p_{i}}{p_{b}} \log \frac{p_{i}}{p_{b}}
\end{gathered}
$$

The sum of two part information entropy is defined as the entropy function: 


$$
H(t)=H_{o}(t)+H_{b}(t)=-\left(\sum_{i=0}^{t} \frac{p_{i}}{p_{o}} \log \frac{p_{i}}{p_{o}}+\sum_{i=t+1}^{L-1} \frac{p_{i}}{p_{b}} \log \frac{p_{i}}{p_{b}}\right)
$$

When the entropy function is maximum, it corresponds to the gray value which is the best threshold[3].

$$
t^{*}=\arg \max H(t) \square \square \square \square \square \square^{0} \leq t \leq t^{-1} \square \square \square \square \square \square \square \square \square \square \square \square \square \square \square
$$

I. The PRINCIPLE OF 2-D MAXIMUM ENTROPY

$\mathrm{Nij}$ is the number of pixels,which is in the region of gray-scale $\mathrm{i}$ and gray mean $\mathrm{j}$. pij is the probability of occurrence for the mean value of $(i, j)[4-5]$.

$$
\begin{aligned}
& p_{i j}=\frac{N_{i j}}{M \times N} \\
& \sum_{i=0}^{L-1} \sum_{j=0}^{L-1} p_{i j}=1 \\
& \sum_{i=0}^{L-1} \sum_{j=0}^{L-1} N_{i j}=M \times N
\end{aligned}
$$

In the type(16): $\mathrm{M} \times \mathrm{N}$ is the size of the image.

In order to make the maximum amount of information for target and background,to determine the threshold in the target region and background region with 2-D maximum entropy method. The target area and background area each have a different probability distribution, the probability of each region is normalized with posteriori probability, in order to enable the regional entropy additive.

If the threshold is $(s, t)$, then

$$
\begin{gathered}
p_{o}=\sum_{i=0}^{s} \sum_{j=0}^{t} p_{i j} \\
p_{b}=\sum_{i=s+1}^{L-1} \sum_{j=t+1}^{L-1} p_{i j} \\
p_{o}+p_{b}=1
\end{gathered}
$$

Defining the discrete 2D entropy is as follows:

$$
H=-\sum_{i=0}^{L-1} \sum_{j=0}^{L-1} p_{i j} \log p_{i j}
$$

the 2D entropy of target region and background region are respectively as follows:

$$
\begin{aligned}
H_{o} & =-\sum_{i=0}^{s} \sum_{j=0}^{t} \frac{p_{i j}}{p_{o}} \log \frac{p_{i j}}{p_{o}}=-\frac{1}{p_{o}}\left(\sum_{i=0}^{s} \sum_{j=0}^{t} p_{i j} \log p_{i j}-\sum_{i=0}^{s} \sum_{j=0}^{t} p_{i j} \log p_{o}\right)=\log p_{o}-\frac{1}{p_{o}} \sum_{i=0}^{s} \sum_{j=0}^{t} p_{i j} \log p_{i j} \\
& =\log p_{o}+\frac{1}{p_{o}} H_{A}
\end{aligned}
$$

In the type(21):

$$
\begin{gathered}
H_{A}=-\sum_{i=0}^{s} \sum_{j=0}^{t} p_{i j} \log p_{i j} \\
H_{b}=-\sum_{i=s+1}^{L-1} \sum_{j=t+1}^{L-1} \frac{p_{i j}}{p_{b}} \log \frac{p_{i j}}{p_{b}} \\
=\log p_{b}+\frac{1}{p_{b}} H_{B}
\end{gathered}
$$$$
=-\frac{1}{p_{b}} \sum_{i=s+1}^{L-1} \sum_{j=t+1}^{L-1} p_{i j} \log p_{i j}+\frac{1}{p_{b}} \sum_{i=s+1}^{L-1} \sum_{j=t+1}^{L-1} p_{i j} \log p_{b}
$$

In the type(23): 


$$
H_{B}=-\sum_{i=s+1}^{L-1} \sum_{j=t+1}^{L-1} p_{i j} \log p_{i j}
$$

Discriminant function of entropy[6-7] is as follows:

$$
H(s, t)=H_{o}+H_{b}
$$

The selection of optimal threshold vector is given as following:

$$
H\left(s^{*}, t^{*}\right)=\max \{H(s, t)\}
$$

\section{The segmentation effect of maximum entropy}

Experiments have been carried on two images using MATLAB7.0, the segmentation results are shown in Figure 1 and Figure 2 and thresholds obtained by these methods are listed in Table I. To demonstrate the good performance of maximum entropy,the segmentation results are obtained by 1D maximum entropy method and 2-D maximum entropy method,which are also used to find the optimal thresholds parameter.
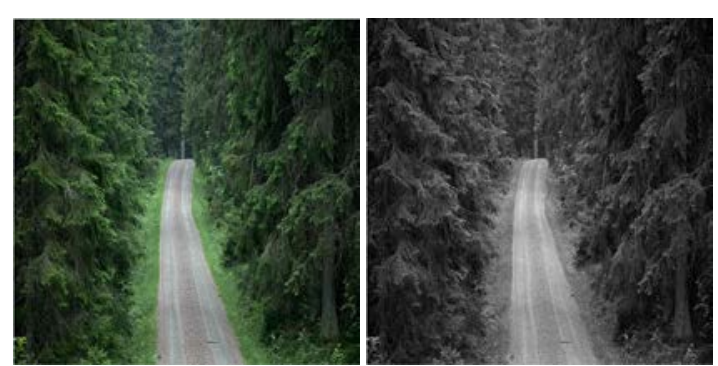

(b)gray image of tree
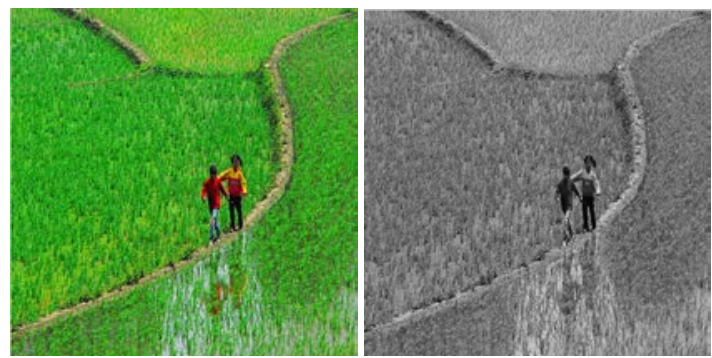

(f)gray image of road

(g) histogram of gray road

(c)histogram of gray tree

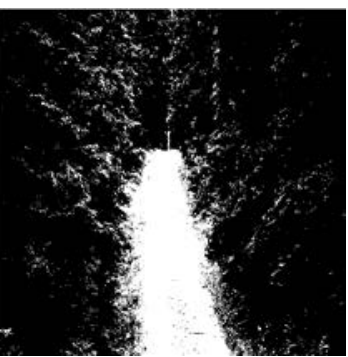

(d)result of tree

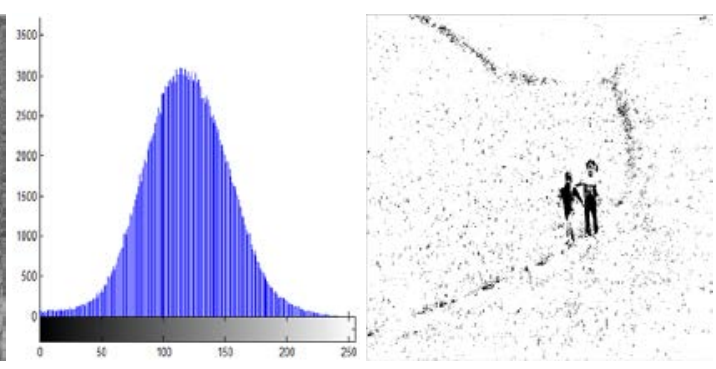

(e)RGB road

Figure1.The segmentation effect of one dimensional maximum entropy
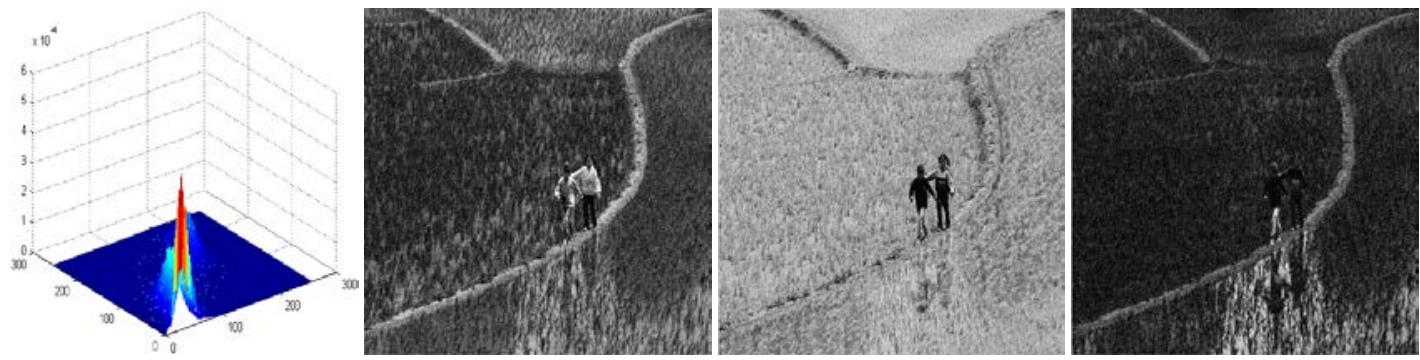

(a) 2-D gray level histogram

(b) R component of road component of road
(d)B 


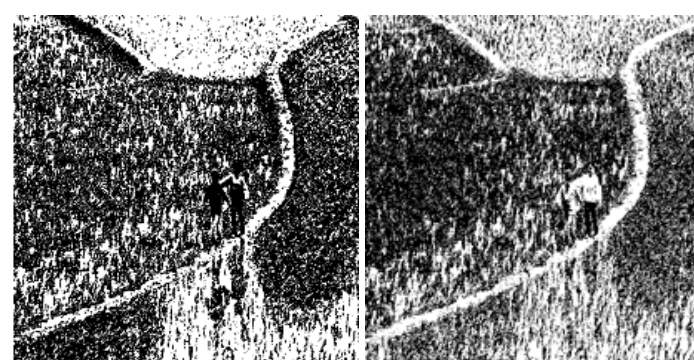

(e) result of gray image of road (f)result of R component result of $\mathrm{B}$ component

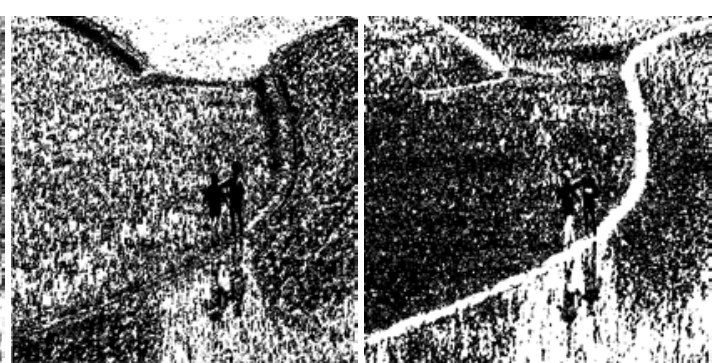

(g)result of G component

(h)

Figure2.The segmentation effect of 2-D maximum entropy

TABLE I. THE PARAMETER OF MAXIMUM ENTROPY

\begin{tabular}{cccccc}
\hline Image & Algorithm & $H_{\max }$ & $s^{*}$ & $t^{*}$ & \multicolumn{2}{c}{ Elapsed time(s) } \\
\hline gray image of tree & 1-D Maximum Entropy & 9.1026 & - & 102 & 0.3900 \\
gray image of road & 1-D Maximum Entropy & 8.6858 & - & 55 & 0.3430 \\
gray image of road & 2-D Maximum Entropy & 15.7624 & 137 & 127 & 2.1370 \\
R component of RGB road & 2-D Maximum Entropy & 16.3259 & 73 & 188 & 2.0130 \\
G component of RGB road & 2-D Maximum Entropy & 15.8254 & 173 & 168 & 2.0900 \\
B component of RGB road & 2-D Maximum Entropy & 15.1524 & 61 & 47 & 1.9810 \\
\hline
\end{tabular}

\section{Conclusion}

This paper proposes a image segmentation method based on maximum entropy. Image segmentation is which extracts objects from background,plays an important role in target recognition. Thresholding is a most used method to segment image for its simplicity and less computation cost. Proposed to use maximum entropy principle to select threshold automatically, used it to image segmentation and enhance.

\section{Acknowledgment}

In this paper, the research was sponsored by the key discipline of Nanchang Normal University, China (Project No.NSXK20141001) and the Reform in Education of Nanchang Normal University, China (Project No.JGKT-14-18).

\section{References}

[1] LU Liang,ZHAO Jing.Fabric defect detection based on wavelet transform and the maximum entropy of images[J].Science Technology and Engineering,11(22):5446-5450

[2] ZHANG Jun-na,FENG Yun-zhi.Image segmentation research based on quantum maximum entropy algorithm[J].Laser \& Infrared,43(5):578-582

[3] Zheng Zhaohui.Radar image segmentation based on maximum entropy[J].Electronic Sci. \& Tech.,21(6):51-53

[4] GUO Juan,YANG Wei-ming,SHI Ya-he. 2-D Maxmium Entropy Method of Image Segmentation Based on Particle Swam Optimization[J].Computer Simulation,22(11):94-96

[5] CHEN Qiu-hong,SHEN Yun-qin.Segmentation based on clustering and maximum entropy method[J]. Computer Simulation,29(1):214-216

[6] ZHANG Hui,GONG Sheng-rong.Image segmentation based on sobel operator and maximum entropy algorithm[J].Computer Science,38(12):278-280

[7] HONG Xia,ZHOU Mu,TIAN Zengshan,DONG Huining.SIFT image matching algorithm based on two-dimensional maximum entropy aided threshold segmentation[J].Semiconductor Optoelectronics,34(4):689-693 